\title{
AN EXTENSION THEOREM FOR CHARACTERS
}

\author{
STEPHEN M. GAGOLA, JR.
}

\begin{abstract}
If $N$ is a normal subgroup of the finite group $G$ and $\psi$ is an irreducible complex character of $N$ that is invariant in $G$, then $\psi$ is extendible to a character of $G$ if $(|G: N|,|N| / \psi(1))=1$.
\end{abstract}

1. Introduction. The theorem mentioned in the abstract may be regarded as a dual version of the following extendibility result: If $N \unlhd G$ and $\psi$ is an irreducible character of $N$ invariant in $G$ satisfying $(|G: N|, o(\operatorname{det} \psi) \psi(1))=1$ then $\psi$ is extendible to an irreducible character of $G$. Here $o(\operatorname{det} \psi)$ denotes the order of the linear character det $\psi$ as an element in the group of linear characters of $N$ (see Corollary 8.16 in [2]). Furthermore, the extension $\chi$ may be chosen so that $(|G: N|, o(\operatorname{det} \chi))=1$, and subject to this extra condition, $\chi$ is unique. No assertion about uniqueness is made concerning the extendibility theorem in the abstract, however.

2. Some needed facts. [2] will be used as a general reference for character theory and [1] is a convenient reference for results in modular representation theory.

(2.1) Let $N \unlhd G$ and let $\psi$ be an irreducible character of $N$ that is invariant in $G$. Assume for every prime divisor $p$ of $|G: N|$ and for every Sylow $p$-subgroup $H / N$ of $G / N$ that $\psi$ extends to a character of $H$. Then $\psi$ extends to a character of $G$.

Proof. See Corollary 11.31 of [2].

(2.2) Let $p$ be a prime and let $\chi$ be an irreducible character of $G$ such that $|G| / \chi(1)$ is prime to $p$. If $B$ is the $p$-block of $G$ containing $\chi$ then $\chi$ is the unique irreducible character of $G$ in $B$. Furthermore, $B$ contains a unique irreducible Brauer character $\varphi$, and $\chi$ lifts $\varphi$.

Proof. See Theorem 62.5 of [1].

(2.3) (Green) Let $N \unlhd G$ with $G / N$ a $p$-group and let $\mu$ be an irreducible Brauer character of $N$ that is invariant in $G$. Then $\mu$ extends to a Brauer character $\varphi$ of $G$.

Proof. Let $F$ be a finite field of characteristic $p$ which is a spliting field for all subgroups of $G$, and let $W$ be an $F[N]$-module affording $\mu$. Choose any composition factor $V$ of $W^{G}$. Since $\mu$ is $G$-invariant we have $V_{N} \simeq e W$ for some positive integer $e$. Now $G$ permutes the finite set of irreducible $F[N]$-submodules of $V_{N}$. By an easy calculation, this set has cardinality

$$
\left(|F|^{e}-1\right) /(|F|-1) \equiv 1 \quad \bmod p \text {. }
$$

Now $N$ is in the kernel of this action, and since $G / N$ is a $p$-group, $G$ must stabilize an irreducible $F[N]$-submodule of $V_{N}$, say $W_{1}$. But then $W_{1}$ is an $F[G]$-submodule

Received by the editors September 29, 1980.

1980 Mathematics Subject Classification. Primary 20C15; Secondary 20 C20.

(C) 1981 American Mathematical Society 0002-9939/81/0000-0405/\$01.50 
of the irreducible module $V$ and hence $W_{1}=V$ and $e=1$. If $\varphi$ is the Brauer character afforded by $V$, then $\varphi$ is an extension of $\mu$, and the result follows.

Notice that in the situation of (2.3), the $p$-regular elements of $G$ are contained in $N$. Viewed as functions then, $\varphi$ is indistinguishable from $\mu$. However, $\varphi$ and $\mu$ are associated with modules over different groups, and so should be viewed as different mathematical objects. This anomaly which arises from the very definition of Brauer character should cause no confusion here.

(2.4) If $\varphi$ is an irreducible Brauer character of $G$ and $q$ is any prime, then there exists an ordinary irreducible character $\chi$ of $G$ whose decomposition number $d_{\chi \varphi}$ is prime to $q$.

Proof. Define the function $\hat{\varphi}$ on $G$ by setting $\hat{\varphi}(x)=\varphi\left(x_{p^{\prime}}\right)$ where $x_{p^{\prime}}$ denotes the $p^{\prime}$-part of $x$. Then $\hat{\varphi}$ is a generalized character of $G$ (Theorem 15.14 of [2]). Write $\hat{\varphi}=\Sigma a_{\chi} \chi$ where the sum extends over all irereducible characters of $G$ and $a_{\chi} \in \mathbf{Z}$. Restricting this equation to $p$-regular elements:

$$
\varphi=\sum_{\chi} a_{\chi} \sum_{\varphi^{\prime}} d_{\chi \varphi^{\prime}} \varphi^{\prime}=\sum_{\varphi^{\prime}}\left(\sum_{\chi} a_{\chi} d_{\chi \varphi^{\prime}}\right) \varphi^{\prime}
$$

By the linear independence of the irreducible Brauer characters we have $\Sigma_{\chi} a_{\chi} d_{x \varphi}$ $=1$. Hence the decomposition numbers $d_{\chi \varphi}$, as $\chi$ ranges over all irreducible characters of $G$, are collectively relatively prime and the assertion follows.

3. Proof of the main theorem. Assume $\psi$ satisfies the hypotheses in the statement of the abstract. By (2.1) we may assume $G / N$ is a $p$-group for some prime $p$. Since $|N| / \psi(1)$ is prime to $p, \psi$ lies in a $p$-block $b$ of defect 0 . By (2.2), b contains a unique irreducible Brauer character $\mu$, and $\psi$ is a lift of $\mu$. Since $\psi$ is invariant in $G$, so is $\mu$, and by (2.3) $\mu$ extends to a Brauer character $\varphi$ of $G$. Now by (2.4), there exists an ordinary irreducible character $\chi$ of $G$ such that $p \nmid d_{\chi \varphi}$.

Let $\chi^{\cdot}$ and $\left(\chi_{N}\right)^{\cdot}$ denote the Brauer characters of $G$ and $N$ associated with $\chi$ and $\chi_{N}$. As $\chi^{\cdot}$ contains $\varphi$ as a constituent and $\varphi_{N}=\mu$, the restriction $\left(\chi^{*}\right)_{N}$ contains $\mu$ as a constituent. But $(\chi)_{N}=\left(\chi_{N}\right)^{\circ}$ and so $\chi_{N}$ contains a constituent $\psi_{0}$ such that $\psi_{0}^{\circ}$ contains $\mu$ as a Brauer constituent. But then $\psi_{0} \in b$ and hence $\psi_{0}=\psi$, since $\psi$ is the unique ordinary irreducible character in $b$. By Clifford's theorem, $\chi_{N}=e \psi$ for some integer $e$ dividing $|G: N|$. Restricting this equation to $p$-singular elements (using $\varphi_{N}=\mu$ again) we have $\chi^{\circ}=e \varphi$ so $e=d_{x \varphi}$. Since $e$ divides $|G: N|, e$ is a power of $p$. However, $p \nmid d_{\chi \varphi}=e$ so $e$ is prime to $p$. Hence $e=1$ and $\chi$ extends $\psi$, as desired.

\section{REFERENCES}

1. L. Dornhoff, Group representation theory, Part B, Marcel Dekker, New York, 1971.

2. I. M. Isaacs, Character theory of finite groups, Academic Press, New York, 1976.

Department of Mathematics, TeXas A\&M University, College Station, TeXas 77843 